\title{
Notes on Some Inequalities for Hilbert Space Operators
}

By

\author{
Fuad KITTANEH*
}

\begin{abstract}
Several inequalities for Hilbert space operators are extended. These include results of Furuta, Halmos, and Kato on the mixed Schwarz inequality, the generalized Reid inequality as proved by Halmos and a classical inequality in the theory of compact non-self-adjoint operators which is essentially due to Weyl. Some related inequalities are also discussed.
\end{abstract}

1980 Mathematics Subject Classification: primary 47A30, 47B10, 37B15.

Key words phrases: mixedSchwarz inequality, Heinz inequality, generalized Reid inequality, Weyl iuequality, Schatten $p$-class.

\section{\$1. Introduction}

Let $H$ be a complex Hilbert space with norm $\|\cdot\|$ and inner product $(.,$.$) .$ Let $B(H)$ denote the algebra of all bounded linear operators on $H ;\|\cdot\|$ will also denote the norm in $B(H)$. The Schwarz inequality for positive operators asserts that if $T$ is a positive operator in $B(H)$, then

$$
|(T x, y)|^{2} \leqslant(T x, x)(T y, y) \quad \text { for all } x, y \text { in } H .
$$

For an arbitrary operator $T$ in $B(H)$, a "mixed Schwarz" inequality has been established in [8] (see also [4] and [6, p. 265].) This inequality asserts that

$$
|(T x, y)|^{2} \leqslant\left(|T|^{2^{\alpha}} x, x\right)\left(\left|T^{*}\right|^{2(1-\alpha)} y, y\right)
$$

for all $x, y$ in $H$ and for $0 \leqslant \alpha \leqslant 1$. Here $|T|=\left(T^{*} T\right)^{1 / 2}$ and $\left|T^{*}\right|=\left(T T^{*}\right)^{1 / 2}$.

An important consequence of (2) is the famous Heinz inequality $[3,7,8]$ which says that if $T, A$, and $B$ are operators in $B(H)$ such that $A$ and $B$ are positive and $\|T x\| \leqslant\|A x\|$ and $\left\|T^{*} y\right\| \leqslant\|B y\|$ for all $x, y$ in $H$, then

Communicated by H. Araki, September 19, 1987.

* Department of Mathematics, Kuwait University, P.O. Box. 5969, Kuwait. 


$$
|(T x, y)| \leqslant\left\|A^{\alpha} x\right\|\left\|B^{1-\alpha} y\right\| \quad \text { for } 0 \leqslant \alpha \leqslant 1 .
$$

The main ingredients in the proofs of (2) given in $[4,6,8]$ are the polar decomposition and elementary aspects of the spectral theorem.

In this paper we present two different proofs of a generalized version of (2). Our proofs rely on the positivity and the self-adjointness of certain operator matrices defined on $H \oplus H$. Thus, these proofs may be considered as new proofs of the Heinz inequality. As applications of (2) we will give a different proof of a classical inequality, which is essentially due to Weyl [12] and an extension of the generalized Reid inequality as proved by Halmos [5, p. 51]. Finally, we use the extension of the generalized Reid inequality to provide an extension of Weyl inequality.

\section{§2. On the Mixed Schwarz Inequality}

In this section we provide two new proofs of a generalized version of (2). Our first proof will be based on three lemmas. We start with a folklore result (see [1, Theorem I.1]) whose proof is included for completeness.

Lemma 1. Let $A, B$, and $C$ be operators in $B(H)$, where $A$ and $B$ are positive. Then $\left[\begin{array}{ll}A & C^{*} \\ C & B\end{array}\right]$ is a positive operator in $B(H \oplus H)$ if and only if $|(C x, y)|^{2} \leqslant$ $(A x, x)(B y, y)$ for all $x, y$ in $H$.

Proof. First assume that $\left[\begin{array}{ll}A & C^{*} \\ C & B\end{array}\right]$ is a positive operator in $B(H \oplus H)$. Then by (1), we have

$$
\left|\left(\left[\begin{array}{ll}
A & C^{*} \\
C & B
\end{array}\right]\left[\begin{array}{l}
x \\
0
\end{array}\right],\left[\begin{array}{l}
0 \\
y
\end{array}\right]\right)\right|^{2} \leqslant\left(\left[\begin{array}{ll}
A & C^{*} \\
C & B
\end{array}\right]\left[\begin{array}{l}
x \\
0
\end{array}\right],\left[\begin{array}{l}
x \\
0
\end{array}\right]\right)\left(\left[\begin{array}{ll}
A & C^{*} \\
C & B
\end{array}\right]\left[\begin{array}{l}
0 \\
y
\end{array}\right],\left[\begin{array}{l}
0 \\
y
\end{array}\right]\right)
$$

for all $x, y$ in $H$. A direct simplification of these inner products now yields (4). Conversely, assume that (4) holds, then for every $x, y$ in $H$,

$$
\begin{aligned}
\left(\left[\begin{array}{ll}
A & C^{*} \\
C & B
\end{array}\right]\left[\begin{array}{l}
x \\
y
\end{array}\right],\left[\begin{array}{l}
x \\
y
\end{array}\right]\right) & =(A x, x)+\left(C^{*} y, x\right)+(C x, y)+(B y, y) \\
& =(A x, x)+(B y, y)+2 \operatorname{Re}(C x, y) \\
& \geqslant 2(A x, x)^{1 / 2}(B y, y)^{1 / 2}+2 \operatorname{Re}(C x, y) \\
& \geqslant 2|(C x, y)|+2 \operatorname{Re}(C x, y) \\
& \geqslant 2|(C x, y)|-2|(C x, y)| \\
& =0 .
\end{aligned}
$$


Hence $\left[\begin{array}{ll}A & C^{*} \\ C & B\end{array}\right]$ is positive and the proof is complete.

Lemma 2. Let $A, B$, and $C$ be operators in $B(H)$ such that $A$ and $B$ are positive and $B C=C A . \quad$ If $\left[\begin{array}{ll}A & C^{*} \\ C & B\end{array}\right]$ is positive in $B(H \oplus H)$, then $\left[\begin{array}{cc}f(A)^{2} & C^{*} \\ C & g(B)^{2}\end{array}\right]$ is also positive, where $f$ and $g$ are non-negative functions on $[0, \infty)$ which are continuous and satisfying the relation $f(t) g(t)=t$ for all $t \in[0, \infty)$.

Proof. Assume first that both $A$ and $B$ are invertible. Since $B C=C A$, it follows that $h(B) C=C h(A)$ for any function that is continuous on $[0, \infty)$. Since $f(t) g(t)=t$ for $t \in[0, \infty)$, it follows that $f(D) g(D)=D$ for any positive operator $D$ in $B(H)$. These two facts now imply that $g(B) B^{-1 / 2} C f(A) A^{-1 / 2}$ $=C$. Consequently,

$$
\left[\begin{array}{cc}
f(A)^{2} & C^{* 2} \\
C & g(B)
\end{array}\right]=\left[\begin{array}{cc}
f(A) A^{2-1 / 2} & 0 \\
0 & g(B) B^{-1 / 2}
\end{array}\right]\left[\begin{array}{cc}
A & C^{*} \\
C & B
\end{array}\right]\left[\begin{array}{cc}
f(A) A^{-1 / 2} & 0 \\
0 & g(B) B^{-1 / 2}
\end{array}\right] .
$$

This relation together with the positivity of $\left[\begin{array}{ll}A & C^{*} \\ C & B\end{array}\right]$ completes the proof in this case. For the general case, apply the argument above to the invertible operators $A_{\varepsilon}=A+\varepsilon$ and $B_{\varepsilon}=B+\varepsilon$ for $\varepsilon>0$ and then let $\varepsilon \rightarrow 0$.

Remark. It should be noticed that in Lemma 2, the assumption $B C=C A$ is essential. To see this consider the operator $S=\left[\begin{array}{ll}4 & 2 \\ 2 & 1\end{array}\right]$ acting on a twodimentional Hilbert space. Then it is obvious that $S$ is positive but $\left[\begin{array}{ll}4^{2 \alpha} & 2 \\ 2 & 1\end{array}\right]$ is not positive for $0<\alpha<\frac{1}{2}$. Here $f(t)=t^{\alpha}$ and $g(t)=t^{1-\alpha}$.

The following lemma, which has been implicity used in [9] is very useful in our first proof of a generalized version of (2).

Lemma 3. If $T$ is an operator in $B(H)$, then $\left[\begin{array}{cc}|T| & T^{*} \\ T & \left|T^{*}\right|\end{array}\right]$ is a positive operator in $B(H \oplus H)$.

Proof. On $H \oplus H$, let $S=\left[\begin{array}{ll}0 & T^{*} \\ T & 0\end{array}\right]$. Then $S$ is self-adjoint and $S^{2}=$ $\left[\begin{array}{cc}T^{*} T & 0 \\ 0 & T T^{*}\end{array}\right]$. By the uniqueness of the square root of a positive operator, it follows that $|S|=\left[\begin{array}{cc}|T| & 0 \\ 0 & \left|T^{*}\right|\end{array}\right]$. Since $S$ is self-adjoint, it follows, by the spectral theorem, that $S+|S|$ is positive. Therefore $\left[\begin{array}{cc}|T| & T^{*} \\ T & \left|T^{*}\right|\end{array}\right]$ is positive 
in $B(H \oplus H)$.

Now we are in a position to present our first proof of a generalized version of (2).

Theorem 1. Let $T$ be an operator in $B(H)$ and let $f$ and $g$ be as in Lemma 2. Then $|(T x, y)| \leqslant\|f(|T|) x\|\left\|g\left(\left|T^{*}\right|\right) y\right\|$ for all $x, y$ in $H$.

Proof. Since $T|T|^{2}=\left|T^{*}\right|^{2} T$, it follows that $T|T|=\left|T^{*}\right| T$. Hence, by Lemmas 2 and 3, we have $\left.]^{f(|T|)^{2}} \begin{array}{cc}T^{*} \\ T & g\left(\left|T^{*}\right|\right)^{2}\end{array}\right]$ is positive in $B(H \oplus H)$. The required inequality now follows from Lemma 1 .

Next we give an alternative proof of Theorem 1, which also uses the polar decomposition. First we begin with a general result.

Theorem 2. Let $V$ be a contraction and $P$ a positive operator in $B(H)$ such that $V P=P V$. Then for $f$ and $g$ as in Lemma 2, we have

$$
|(V P x, y)| \leqslant\|f(P) x\|\|g(P) y\| \quad \text { for all } x, y \text { in } H .
$$

Proof. We have

$$
\begin{aligned}
|(V P x, y)| & =\left|\left(P x, V^{*} y\right)\right| \\
& \left.=\left|\left(f(P) x, g(P) V^{*} y\right)\right| \text { (because } P=f(P) g(P)\right) \\
& \left.=\left|\left(f(P) x, V^{*} g(P) y\right)\right| \text { (because } V^{*} g(P)=g(P) V^{*}\right) \\
& \leqslant|f(P) x|\||| g(P) y\| \text { (because } V \text { is a contraction). }
\end{aligned}
$$

Corollary 1. If $T$ is a self-adjoint operator in $B(H)$, $f$ and $g$ are as in Lemma 2 , then $|(T x, y)| \leqslant\|f(|T|) x\|\|g(|T|) y\|$ for all $x, y$ in $H$.

Proof. Let $T=U|T|$ be the polar decomposition of $T$. Then $|T| U=$ $U|T|$ and $U$ is unitary. Now the result follows from Theorem 2.

After we have proved Theorem 1 for self-adjoint operators, we can extend it to arbitrary operators as follows: Let $T, f, g, x$, and $y$ be as in Theorem 1 . Then $S=\left[\begin{array}{cc}0 & T^{*} \\ T & 0\end{array}\right]$ defined in $B(H \oplus H)$ is self-adjoint and $|S|=\left[\begin{array}{cc}|T| & 0 \\ 0 & \left|T^{*}\right|\end{array}\right]$. Moreover, $f(|S|)=\left[\begin{array}{cc}f(|T|) & 0 \\ 0 & f\left(\left|T^{*}\right|\right)\end{array}\right]$ and $g(|S|)=\left[\begin{array}{cc}g(|T|) & 0 \\ 0 & g\left(\left|T^{*}\right|\right)\end{array}\right]$. Applying Corollary 1 to the operator $S$, we see that $|(S u, v)| \leqslant\|f(|S|) u\|\|g(|S|) v\|$ for all $u, v$ in $H \oplus H$. Let $u=\left[\begin{array}{l}x \\ 0\end{array}\right]$ and $v=\left[\begin{array}{l}0 \\ y\end{array}\right]$. Then $(S u, v)=(T x, y)$ and $\|f(|S|) u\|=\|f(|T|) x\|$ and $\|g(|S|) v\|=\left\|g\left(\left|T^{*}\right|\right) y\right\|$. Hence $|(T x, y)| \leqslant\|f(|T|) x\|$ 
$\left\|g\left(\left|T^{*}\right|\right) y\right\|$ for all $x, y$ in $H$ and this completes the second proof of Theorem 1.

Remark. The mixed Schwarz inequality (2) becomes a special case of inequality (5) upon letting $f(t)=t^{\alpha}$ and $g(t)=t^{1-\alpha}$, where $t \in[0, \infty)$ and $0 \leqslant \alpha \leqslant 1$.

Our proofs of Theorem 1 lead to new simplified proofs of the following generalized Heinz inequality, which has been proved for unbounded operators by Kato [8]. First recall that a real valued continuous function $f$ on $[0, \infty)$ is said to be operator monotone if $f(A) \leqslant f(B)$ whenever $0 \leqslant A \leqslant B$.

Corollary 2. Let $T, A$, and $B$ be operators in $B(H)$ such that $A$ and $B$ are positive and $\|T x\| \leqslant\|A x\|$ and $\left\|T^{*} y\right\| \leqslant\|B y\|$ for all $x, y$ in $H$. Let $f$ and $g$ be non-negative operator monotone functions on $[0, \infty)$ such that $f(t) g(t)=t$ for all $t \in[0, \infty)$. Then

$$
|(T x, y)| \leqslant\|f(A) x\|\|g(B) y\| \quad \text { for all } x, y \text { in } H .
$$

For a self-adjoint operator $T$ in $B(H)$, it follows by the spectral theorem that $-|T| \leqslant T \leqslant|T|$ or, equivalently,

$$
|(T x, x)| \leqslant(|T| x, x) \quad \text { for all } x \text { in } \mathrm{H} \text {. }
$$

This inequality can be extended to hyponormal operators as follows.

Theorem 3. Let $T$ be a hyponormal operator in $B(H)$; that is, $T T^{*} \leqslant T^{*} T$. If $f$ and $g$ are as in Corollary 2, then

$$
|(T x, y)| \leqslant\|f(|T|) x\|\|g(|T|) y\| \quad \text { for all } x, y \text { in } H .
$$

In particular $\left(f(t)=g(t)=t^{1 / 2}\right),|(T x, x)| \leqslant(|T| x, x)$ for all $x$ in $H$.

Proof. It is known (see e.g. [2, Corollary 4.3]) that if $h$ is a non-negative operator monotone function on $[0, \infty)$, then $h\left(t^{1 / 2}\right)^{2}$ is also operator monotone. This together with the hyponormality of $T$ implies that $\left\|h\left(\left|T^{*}\right|\right) x\right\| \leqslant$ $\|h(|T|) x\|$ for all $x$ in $H$. The desired conclusion now follows from Theorem 1 .

Remark. Inequality (7) is not true for arbitrary operators. For example, let $T=\left[\begin{array}{ll}0 & 1 \\ 0 & 0\end{array}\right], x=\left[\begin{array}{l}2 \\ 1\end{array}\right]$. Then $|(T x, x)|=2$ but $(|T| x, x)=1$.

\section{§3. On the Weyl Inequality}

As an application of a special case $\left(\alpha=\frac{1}{2}\right)$ of (2), we give a new proof of a basic inequality due to Weyl, which is frequently used in the theory of compact non-self-adjoint operators (see [12, Theorem 2.3] and the references given there). 
Recall that a compact operator $T$ in $B(H)$ is said to be in the Schatten $p$-class $C_{p}(H)(1 \leqslant p<\infty)$, if trace $|T|^{p}<\infty$. The Schatten $p$-norm of $T$ is defined by $\|T\|_{p}=\left(\operatorname{trace}|T|^{p}\right)^{1 / p}$. It is well known that for $T$ in $C_{p}(H),\|T\|_{p}=\left\|T^{*}\right\|_{p}=$ $\||| T \mid\|_{p}$. It is evident by Lemma 1 that if $A, B$, and $C$ are operators in $B(H)$, where $A$ and $B$ are positive such that $\left[\begin{array}{ll}A & C^{*} \\ C & B\end{array}\right]$ is a positive operator in $B(H \oplus H)$, then

$$
\|C\|^{2} \leqslant\|A\|\|B\| \text {. }
$$

An analogous result which is valid for the Schatten $p$-norm can be proved. First we need to recall (see [13, p. 20]) that if $A$ is a positive operator in $B(H)$ and $x$ is any unit vector in $H$, then

$$
(A x, x)^{p} \leqslant\left(A^{p} x, x\right) \quad \text { for } 1 \leqslant p<\infty .
$$

This inequality is essential for us to accomplish our goal. Here is a proof of it: Let $A=\int_{0}^{\infty} t d E(t)$ be the spectral representation of $A$. Then $(A x, x)^{p}=$ $\left(\int_{0}^{\infty} t d(E(t) x, x)\right)^{p} \leqslant \int_{0}^{\infty} t^{p} d(E(t) x, x)=\left(A^{p} x, x\right)$. Here we have used Jensen's inequality applied to the convex function $f(t)=t^{p}$ defined on $[0, \infty)$.

Theorem 4. Let $A$ and $B$ be positive operators in $C_{p}(H)$ and in $C_{q}(H)$, respectively, where $1 \leqslant p, q<\infty$. Let $C$ be an operator in $B(H)$ such that $\left[\begin{array}{ll}A & C^{*} \\ C & B\end{array}\right]$ is a positive operator in $B(H \oplus H)$. Then for any orthonormal sets $\left\{x_{n}\right\},\left\{y_{n}\right\}$ in $H$, we have

$$
\sum_{n}\left|\left(C x_{n}, y_{n}\right)\right|^{2 r} \leqslant\|A\|_{p}^{r}\|B\|_{q}^{r}, \quad \text { where } \frac{1}{r}=\frac{1}{p}+\frac{1}{q} .
$$

Proof. Since $\left[\begin{array}{ll}A & C^{*} \\ C & B\end{array}\right]$ is a positive operator in $B(H \oplus H)$, it follows by Lemma 1, that $\left|\left(C x_{n}, y_{n}\right)\right|^{2 r} \leqslant\left(A x_{n}, x_{n}\right)^{r}\left(B y_{n}, y_{n}\right)^{r}$ for all $n$. Summing over $n$ and using Holder's inequality, we obtain that

$$
\begin{aligned}
\sum_{n}\left|\left(C x_{n}, y_{n}\right)\right|^{2 r} & \leqslant \sum_{n}\left(A x_{n}, x_{n}\right)^{r}\left(B y_{n}, y_{n}\right)^{r} \\
& \leqslant\left(\sum_{n}\left(A x_{n}, x_{n}\right)^{p}\right)^{r / p}\left(\sum_{n}\left(B y_{n}, y_{n}\right)^{q}\right)^{r / q} \\
& \leqslant\left(\sum_{n}\left(A^{p} x_{n}, x_{n}\right)\right)^{r / p}\left(\sum_{n}\left(B^{q} y_{n}, y_{n}\right)\right)^{r / q} \quad \text { by (10) } \\
& \leqslant\left(\operatorname{trace} A^{p}\right)^{r / p}\left(\operatorname{trace} B^{q}\right)^{r / q} \\
& =\|A\|_{p}^{r}\|B\|_{q}^{r} \text { as required. }
\end{aligned}
$$

Letting $p=q$, in Theorem 4 , enables us to give the following alternative 
proof of Theorem 2.3 in [12], which is the desired application of (2).

Corollary 3 (Weyl's inequality). Let $T$ be an operator in $C_{p}(H)$, where $1 \leqslant p<\infty$. Then for any orthonormal sets $\left\{x_{n}\right\},\{y\}_{n}$ in $H$, we have

$$
\sum_{n}\left|\left(T x_{n}, y_{n}\right)\right|^{p} \leqslant\|T\|_{p}^{p}
$$

Proof. Since $\left[\begin{array}{cc}|T| & T^{*} \\ T & \left|T^{*}\right|\end{array}\right]$ is a positive operator in $B(H \oplus H)$ or, equivalently by (2) $\left(\alpha=\frac{1}{2}\right)$, it follows from Theorem 4 that $\sum_{n}\left|\left(T x_{n}, y_{n}\right)\right|^{p} \leqslant$ $\||T|\|_{p}^{p / 2}\left\|\left|T^{*}\right|\right\|_{p}^{p / 2}=\|T\|_{p}^{p}$ as required.

Regarding Corollary 3, it is, in fact, well known (see [11] or [13]), that if $T$ is an operator in $C_{p}(H)$, where $1 \leqslant p<\infty$, then

$$
\|T\|_{p}^{p}=\sup \sum_{n}\left|\left(T x_{n}, y_{n}\right)\right|^{p},
$$

where the supremum is taken over all orthonormal sets $\left\{x_{n}\right\},\left\{y_{n}\right\}$ in $H$.

Using (13) and Theorem 4, we have the following inequality which is analogous to (9).

Corollary 4. Let $A$ and $B$ be positive operators in $C_{p}(H)$ and in $C_{q}(H)$, respectively, where $1 \leqslant p, q<\infty$. Let $C$ be an operator in $B(H)$ such that $\left[\begin{array}{ll}A & C^{*} \\ C & B\end{array}\right]$ is a positive operator in $B(H \oplus H)$. Then $C$ belongs to $C_{2 r}$, where $\frac{1}{r}=\frac{1}{p}+\frac{1}{q}$, and

$$
\|C\|_{2 r}^{2} \leqslant\|A\|_{p}\|B\|_{q}
$$

Remark. If, in Corollary 4, we assume that $p=q$, that is, both $A$ and $B$ are in $C_{p}(H)$, then $C$ is also in $C_{p}(H)$, and in this case

$$
\|C\|_{p}^{2} \leqslant\|A\|_{p}\|B\|_{p} .
$$

However, if we merely assume that either $A$ or $B$ is in $C_{p}(H)$, then $C$ belongs to $C_{2 p}(H)$. In fact, it follows easily from (13) and the proof of Theorem 4 that if $A$ is in $C_{p}(H)$, then

$$
\|C\|_{2 p}^{2} \leqslant\|A\|_{p}\|B\|
$$

On the other hand, if $B$ is in $C_{p}(H)$, then

$$
\|C\|_{2 p}^{2} \leqslant\|A\|\|B\|_{p} .
$$

In the spirit of this remark, we conclude this section with the following 
result concerning the compactness of $C$.

Corollary 5. Let $A$ and $B$ be positive operators in $B(H)$, where either $A$ or $B$ is compact. Let $C$ be an operator in $B(H)$ such that $\left[\begin{array}{ll}A & C^{*} \\ C & B\end{array}\right]$ is a positive operator in $B(H \oplus H)$. Then $C$ is compact.

Proof. This is a consequence of Lemma 1 and the fact [11, p. 58], that an operator $T$ in $B(H)$ is compact if and only if $\left(T x_{n}, x_{n}\right) \rightarrow 0$ as $n \rightarrow \infty$ for every orthonormal set $\left\{x_{n}\right\}$ in $H$.

\section{§4. On the Generalized Reid Inequality}

The generalized Reid inequality as proved by Halmos [5] asserts that if $A$ and $B$ are operators in $B(H)$ such that $A$ is positive and $A B$ is self-adjoint, then

$$
|(A B x, x)| \leqslant r(B)(A x, x) \quad \text { for all } x \text { in } H .
$$

Here $r(B)$ denotes the spectral radius of $B$. A weaker version of this inequality was proved by Reid [10] in which $r(B)$ is replaced by $\|B\|$.

In this section we establish an inequality which extends both the generalized Reid inequality (18) and the generalized mixed Schwarz inequality (Theorem 1) and then we will use a special case of this inequality to extend Weyl's inequality.

Theorem 5. Let $A$ and $B$ be operators in $B(H)$ such that $|A| B=B^{*}|A|$. If $f$ and $g$ are as in Lemma 2, then

$$
|(A B x, y)| \leqslant r(B)\left\|f ( | A | ) x \left|\left\||| g\left(\left|A^{*}\right|\right) y\right\| \quad \text { for all } x, y \text { in } H .\right.\right.
$$

Proof. The main idea in the proof is to establish the following inequality:

$$
|(A B x, y)|^{2^{n}} \leqslant\left(f(|A|)^{2} B^{2^{n}} x, x\right)\left(f(|A|)^{2} x, x\right)^{2^{n-1}-1}\left(g\left(\left|A^{*}\right|\right)^{2} y, y\right)^{2^{n-1}} .
$$

This can be proved by induction. First we prove it for $n=1$. Now

$$
\begin{aligned}
|(A B x, y)|^{2} & \leqslant\|f(|A|) B x\|^{2}\left\|g\left(\left|A^{*}\right|\right) y\right\|^{2} \quad \text { (by Theorem 1) } \\
& =(f(|A|) B x, f(|A|) B x)\left(g\left(\left|A^{*}\right|\right) y, g\left(\left|A^{*}\right|\right) y\right) \\
& =\left(B^{*} f(|A|)^{2} B x, x\right)\left(g\left(\left|A^{*}\right|\right)^{2} y, y\right) \\
& \left.=\left(f(|A|)^{2} B^{2} x, x\right)\left(g\left(\left|A^{*}\right|\right)^{2} y, y\right) \text { (because } B^{*} f(|A|)^{2}=f(|A|)^{2} B\right),
\end{aligned}
$$

and so (20) is true for $n=1$. Assume that (20) is true for some integer $n>1$. Then

$$
\begin{aligned}
|(A B x, y)|^{2^{n+1}} & =\left(|(A B x, y)|^{2^{n}}\right)^{2} \\
\leqslant & {\left[\left(f(|A|)^{2} B^{2^{n}} x, x\right)\left(f(|A|)^{2} x, x\right)^{2^{n-1}-1}\left(g\left(\left|A^{*}\right|\right)^{2} y, y\right)^{2^{n-1}}\right]^{2} }
\end{aligned}
$$




$$
\begin{aligned}
& \leqslant\left(f(|A|)^{2} B^{2^{n}} x, B^{2^{n}} x\right)\left(f(|A|)^{2} x, x\right)\left(f(|A|)^{2} x, x\right)^{2^{n}-2}\left(g\left(\left|A^{*}\right|\right)^{2} y, y\right)^{2^{n}} \\
& =\left(B^{* 2^{n}} f(|A|)^{2} B^{2^{n}} x, x\right)\left(f(|A|)^{2} x, x\right)^{2^{n}-1}\left(g\left(\left|A^{*}\right|\right)^{2} y, y\right)^{2^{n}} \\
& =\left(f(|A|)^{2} B^{2^{n+1}} x, x\right)\left(f(|A|)^{2} x, x\right)^{2^{n}-1}\left(g\left(\left|A^{*}\right|\right)^{2} y, y\right)^{2^{n}}
\end{aligned}
$$

which proves (20).

Notice that we have used (1) in the second inequality and the fact $B^{* 2^{n}} f(|A|)^{2}=$ $f(|A|)^{2} B^{2^{n}}$ in the last equation above. Now, as in the proof of the generalized Reid inequality [5], we have from (20) that

$$
|(A B x, y)|^{2^{n}} \leqslant\left\|f(|A|)^{2}\right\|\left\|B^{2^{n}}\right\|\|x\|^{2}\left(f(|A|)^{2} x, x\right)^{2^{n-1}-1}\left(g\left(\left|A^{*}\right|\right)^{2} y, y\right)^{2^{n-1}}
$$

and so

$$
|(A B x, y)| \leqslant\left.\left\|\left.f(|A|)^{2}\right|^{1 / 2^{n}}\right\| B^{2^{n}}||^{1 / 2^{n}}|| x\right|^{2 / 2^{n}}\left(f(|A|)^{2} x, x\right)^{(1 / 2)-\left(1 / 2^{n}\right)}\left(g\left(\left|A^{*}\right|\right)^{2} y, y\right)^{1 / 2} .
$$

Letting $n \rightarrow \infty$, we obtain $|(A B x, y)| \leqslant r(B)\|f(|A|) x \mid\|\left\|g\left(\left|A^{*}\right|\right) y\right\|$.

Remarks (1). If we let $B=1$ (the identity operator) in Theorem 5 , then we retain Theorem 1 .

(2) If in Theorem $5, A$ is assumed to be positive, then the condition $A B=B^{*} A$ is equivalent to saying that $A B$ is self-adjoint. In this case, letting $f(t)=g(t)=t^{1 / 2}$ and $x=y$, we obtain the generalized Reid inequality (18) as a special case.

Using Theorem 5 (the case $f(t)=g(t)=t^{1 / 2}$ ) and following the proof of Corollary 3 , enable us to obtain the following inequality which we may call a generalized Weyl inequality.

Corollary 6. Let $A$ be an operator in $C_{p}(H)$ and let $B$ be an operator in $B(H)$ such that $|A| B=B^{*}|A|$. Then for any orthonormal sets $\left\{x_{n}\right\},\left\{y_{n}\right\}$ in $H$ we have

$$
\sum_{n}\left|\left(A B x_{n}, y_{n}\right)\right|^{p} \leqslant r(B)^{p}\|A\|_{p}^{p}
$$

Moreover,

$$
\|A B\|_{p} \leqslant r(B)\|A\|_{p} .
$$

Proof. Notice that we have $\left|\left(A B x_{n}, y_{n}\right)\right|^{2} \leqslant r(B)^{2}\left(|A| x_{n}, x_{n}\right)\left(\left|A^{*}\right| y_{n}, y_{n}\right)$ and then proceed as in the proofs of Theorem 4 and Corollary 3. The last assertion follows from (13).

Another extension of the mixed Schwarz inequality (2), which is related to the generalized Reid inequality (18), is the following. 
Theorem 6. Let $A_{1}, A_{2}, \cdots$, and $A_{n}$ be operators in $B(H)$, where $n \geqslant 2$. Then

$$
\left|\left(\left(A_{1} A_{2} \cdots A_{n}\right) x, y\right)\right|^{2} \leqslant a_{1}^{2 \alpha} a_{n}^{2(1-\alpha)} a_{2}^{2} \cdots a_{n-1}^{2}\left(\left|A_{n}\right|^{2^{\alpha}} x, x\right)\left(\left|A_{1}^{*}\right|^{2(1-\alpha)} y, y\right)
$$

for all $x, y$ in $H$ and $0 \leqslant \alpha \leqslant 1$, where $a_{i}=\left\|A_{i}\right\|$.

Proof. If $n=2$, then $\left|\left(A_{1} A_{2} x, y\right)\right|^{2} \leqslant\left(\left|A_{1} A_{2}\right|^{2 \alpha} x, x\right)\left(\left|A_{2}^{*} A_{1}^{*}\right|^{2(1-\alpha)} y, y\right)$ by (2). But $\left|A_{1} A_{2}\right|^{2}=A_{2}^{*} A_{1}^{*} A_{1} A_{2} \leqslant a_{1}^{2}\left|A_{2}\right|^{2}$. Hence $\left|A_{1} A_{2}\right|^{2^{\alpha}} \leqslant a_{1}^{2 \alpha}\left|A_{2}\right|^{2^{\alpha}}$. Similarly $\left|A_{2}^{*} A_{1}^{*}\right|^{2(1-\infty)} \leqslant a_{2}^{2) 1-\infty)}\left|A_{1}^{*}\right|^{2(1-\alpha)}$. Here we have used the fact that the function $f(t)=t^{\alpha}$ is operator monotone on $[0, \infty)$, where $0 \leqslant \alpha \leqslant 1$. Thus (23) is true for $n=2$. If $n>2$, then by the previous case,

$$
\begin{aligned}
& \left|\left(\left(A_{1} A_{2} \cdots A_{n}\right) x, y\right)\right|^{2}=\left|\left(\left(A_{1} A_{2} \cdots A_{n-1}\right) A_{n} x, y\right)\right|^{2} \\
& \quad \leqslant|| A_{1} A_{2} \cdots A_{n-1}||^{2 \alpha} a_{n}^{2(1-\alpha)}\left(\left|A_{n}\right|^{2 \alpha} x, x\right)\left(\left|A_{n-1}^{*} \cdots A_{1}^{*} A_{2}^{*}\right|^{2(1-\alpha)} y, y\right) \\
& \quad \leqslant a_{1}^{2 \alpha} a_{2}^{2 \alpha} \cdots a_{n-1}^{2 \alpha} a_{n}^{2(1-\alpha)}\left(\left|A_{n}\right|^{2 \alpha} x, x\right)\left(\left|A_{n-1}^{*} \cdots A_{1}^{*} A_{2}^{*}\right|^{2(1-\alpha)} y, y\right) .
\end{aligned}
$$

But $\left|A_{n-1}^{*} \cdots A_{2}^{*} A_{1}^{*}\right|^{2} \leqslant a_{2}^{2} a_{3}^{2} \cdots a_{n-1}^{2}\left|A_{1}^{*}\right|^{2}$. Hence

$$
\left|A_{n-1}^{*} \cdots A_{2}^{*} A_{1}^{*}\right|^{2(1-\alpha)} \leqslant a_{2}^{2(1-\alpha)} a_{3}^{2(1-\alpha)} \cdots a_{n-1}^{2(1-\alpha)}\left|A_{1}^{*}\right|^{2(1-\alpha)} \quad \text { and so }
$$

$\left|\left(\left(A_{1} A_{2} \cdots A_{n}\right)\right) x, y\right|^{2} \leqslant a_{1}^{2 \alpha} a_{n}^{2(1-\alpha)} a_{2}^{2} \cdots a_{n-1}^{2}\left(\left|A_{n}\right|^{2 \alpha} x, x\right)\left(\left|A_{1}^{*}\right|^{2(1-\alpha)} y, y\right)$, which completes the proof.

Corollary 7. Let $A$ be an operator in $B(H)$. Then for any integer $n \geqslant 1$,

$$
\left|\left(A^{n} x, y\right)\right|^{2} \leqslant\|A\|^{2 n-2}\left(|A|^{2^{\alpha}} x, x\right)\left(\left|A^{*}\right|^{2(1-\alpha)} y, y\right) \quad \text { for all } x, y
$$

in $H$ and $0 \leqslant \alpha \leqslant 1$.

We would like to conclude with the following inequality which is related to (24). The proof, which can be completed by induction and (1), is omitted.

Theorem 7. If $A$ is an operator in $B(H)$, then

$$
\|A x\|^{2^{n-1}+2} \leqslant(|A| x, x)^{2^{n-2}}\left(|A|^{2^{n-2}+2} x, x\right) \quad \text { for all } x \text { in } H \text { and } n=2,3, \cdots
$$

Using (10) we have the following corollary.

Corollary 8. If $A$ is an operator in $B(H)$, then

$\| A x||^{2^{n}+2} \leqslant\left(|A|^{2^{n}+2} x, x\right)$ for any unit vector $x$ in $H$ and any integer $n \geqslant 0$.

\section{References}

[1] Ando, T., Topics on operator inequalities, Preprint, Research Institute of Applied Electricity, Hokkaido University, Sapporo, Japan. 
[2] Ando, T., Concavity of certain maps on positive definite matrices and applications to Hadamard products, Linear Algebra and Appl. 26, (1979), 203-241.

[3] Dixmier, J., Sur une ineqalite de E. Heinz, Math. Ann., 126 (1953), 75-78.

[4] Furuta, T., A simplified proof of Heinz inequality and scrutiny of its equality, Proc. Amer. Math. Soc., 97 (1986), 751-753.

[5] Halmos, P.R., A Hilbert space problem book, Van Nostrand Company, Inc., Princeton, N.J., 1967.

[6] - A Hilbert space problem book, Springer Verlag, New York, 1982.

[7] Heinz, E., On an inequality for linear operators in Hilbert space, Report on Operator Theory and Group Representation, Publ. No. 387, Nat. Acad. Sci.-Nat. Res. Council, Washington D.C., 1955, pp. 27-29.

[8] Kato, T., Notes on some inequalities for linear operators, Math. Ann., 125 (1952), 208-212.

[9] Kittaneh, F., Inequalities for the Schatten p-norm. IV, Commun. Math., Phys. 106 (1986), 581-585.

[10] Reid, W., Symmetrizable completely continuous linear tarnsformations in Hilbert space, Duke Math. J., 18 (1951), 41-56.

[11] Ringrose, J.R., Compact non-self-adjoint operators, Van Nostrand Reinhold, New York, 1971.

[12] Simon, B., Notes on infinite determinants of Hilbert space operators, Adv. Math., 24 (1977), 244-273.

[13] — , Trace ideals and their applications, Camrbidge University Press, 1979. 
